\section{Syringocystadenoma papilliferum und Basalzellkarzinom der Kopfhaut}

Zusammenfassung. Das Syringocystadenoma papilliferum (SCAP) ist ein benigner Schweißdrüsentumor, der seit Geburt oder früher Kindheit besteht und am häufigsten auf dem Kapillitium lokalisiert ist. In ca. $\mathbf{3 0} \%$ der Fälle entwickelt sich das SCAP in einem Naevus sebaceus Jadassohn und kann dabei auch noch mit weiteren Neubildungen vergesellschaftet sein, u.a. mit einem Basalzellkarzinom. Demgegenüber ist die Koinzidenz eines SCAP mit einem Basalzellkarzinom ohne Naevus sebaceus, wie von uns bei einer 64-jährigen Frau diagnostiziert, selten. Die Therapie der Wahl eines SCAP ist die Exzision mit ausreichendem Sicherheitsabstand.

Syringocystadenoma Papilliferum and Basal Cell Carcinoma of the Scalp. Syringocystadenoma papilliferum (SCAP) is a benign tumour of the sweat glands that exists as a „birthmark“ or from early childhood. Most frequently it is localised on the scalp. Up to $30 \%$ the SCAP develops in a naevus sebaceus of Jadassohn. In such cases it can be associated with other neoplasms, also with a basal cell carcinoma. On the other hand, coincidence of SCAP and basal cell carcinoma without a naevus sebaceus as observed by us in a 64-year old female is rare. The therapy of choice ist complete surgical excision observing sufficiently safe resection margins.

\section{Einleitung}

Das Syringocystadenoma papilliferum (SCAP) wurde erstmals von Elliot 1893 [5] unter dem Begriff Adenocystoma intra canaliculare beschrieben. Die heute gebräuchliche Bezeichnung SCAP geht auf eine Mitteilung von Werther 1913 zurück [27]. Es handelt sich um einen benignen Schweißdrüsentumor, der sich bei Geburt oder in früher Kindheit manifestiert und eine langsame Wachstumstendenz aufweist. Nicht selten tritt das SCAP in einem Naevus sebaceus Jadassohn [7] auf, dabei gelegentlich kombiniert mit weiteren Neubildungen. Die Koinzidenz eines SCAP mit einem Basalzellkarzinom ohne Naevus sebaceus ist unseres Wissens weitaus weniger häufig und veranlasst uns zu nachfolgender Darstellung.

Akt Dermatol 2001; 27: 70-73

(c) Georg Thieme Verlag Stuttgart · New York ISSN 0340-2541
M. Wiesner ${ }^{1}$, C. Woenckhaus², K. Hein', T. Dittberner ${ }^{1}$

${ }^{1}$ Klinik und Poliklinik für Hautkrankheiten (Komm. Direktor: OA Dr. M. Wiesner)

2 Institut für Pathologie (Direktor: Prof. Dr. G. Lorenz) der Ernst-Moritz-Arndt-Universität Greifswald

\section{Fallbericht}

\section{Anamnese}

Bei einer 64-jährigen Patientin bestand seit der Geburt ein „Muttermal“ auf dem behaarten Kopf. Seit 2 Jahren würde dieser Herd hin und wieder jucken, nässen und auch bluten.

\section{Aufnahmebefund}

Parietal links befindet sich eine im Durchmesser $10 \mathrm{~mm}$ große gelbliche flach erhabene, teils papillomatöse, im vorderen Anteil oberflächlich ulzerierte Plaque (Abb.1). Auf Druck entleert sich etwas dünnflüssiges Sekret.

\section{Histologie}

In dem exzidierten Tumor sind eng beieinanderliegend zwei histomorphologisch unterschiedliche Anteile zu erkennen. Auf der einen Seite handelt es sich um ein zystisches Gebilde mit hineinragenden plumpen zottenartigen Vorsprüngen, die von einem zweireihigen Epithel bedeckt sind (Abb. 2). Dessen lumenwärts gerichtete zylindrische Zellen weisen eine deutliche Sekretion auf (Abb. 3). Das Tumorstroma wird vorwiegend von Lymphozyten, aber auch von einzelnen Plasmazellen durchsetzt. In unmittelbarer Nachbarschaft finden sich von der Epidermis ausgehende, verzweigte und scharf begrenzte Stränge basaloider Zellen, die in den äußeren Lagen pallisadenartig angeordnet sind und pleomorphe sowie pyknotische Zellkerne mit vermehrten Mitosen aufweisen (Abb. 2, 4). Diese Zellen lassen sich nicht mit dem Antikörper gegen Neuronspezifische Enolase markieren. Merkmale einer follikulären Differenzierung wie rudimentäre follikuläre Keime und Papillen sind auch nach vollständiger Aufarbeitung des Präparates in Stufenschnitten nicht nachzuweisen. Anteile eines Naevus sebaceus kommen dabei ebenfalls nicht zur Darstellung. 


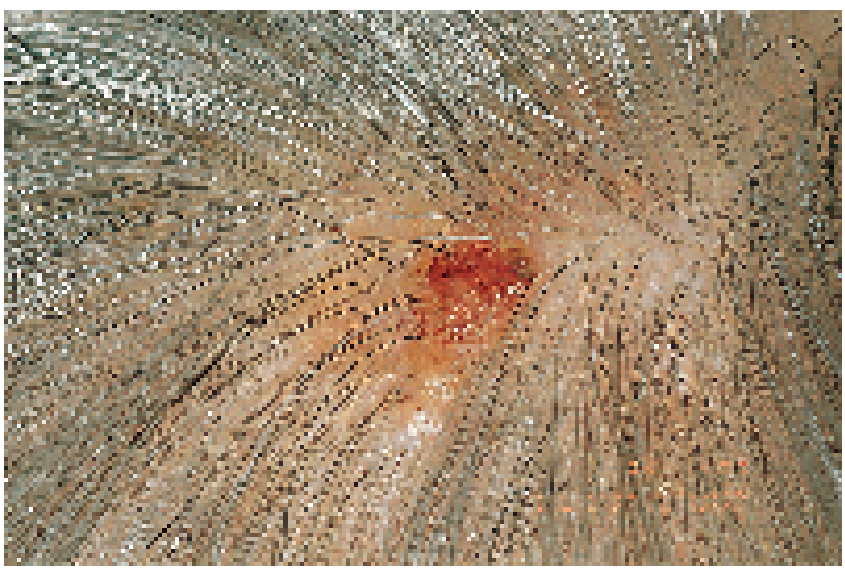

Abb.1 SCAP. Eine gelbliche, im vorderen Anteil oberflächlich ulzerierte Plaque auf dem Kapillitium.

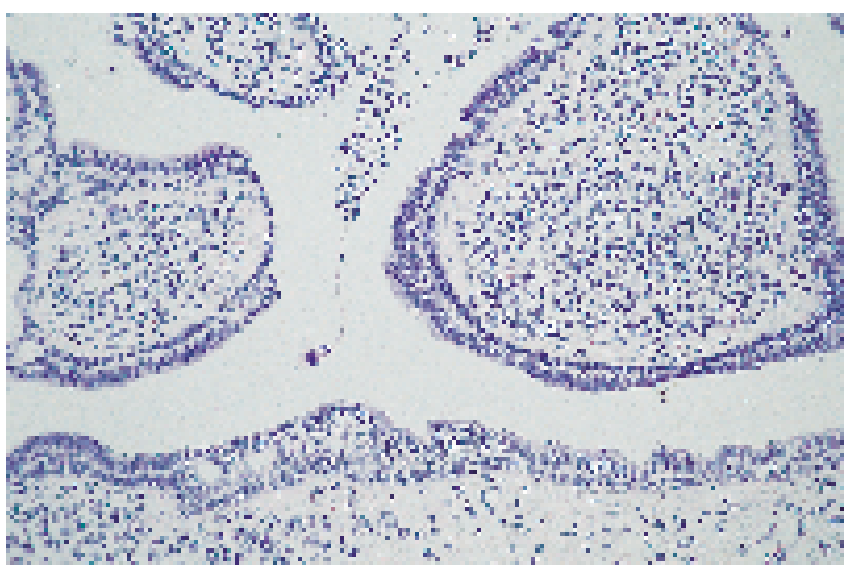

Abb.3 SCAP. Zweireihiges, lumenwärts zylindrisches Zystenepithel, HE x 250 .

\section{Diagnose}

Syringocystadenoma papilliferum kombiniert mit einem oberflächlichen soliden Basalzellkarzinom.

\section{Therapie und Verlauf}

Der Tumor wurde in Lokalanästhesie in toto exzidiert; postoperativ traten keine Komplikationen auf.

\section{Diskussion}

Das SCAP präsentiert sich klinisch als gelblich-rötlicher bis rötlich-bräunlicher haarloser plaqueförmiger, kleinknotiger oder papillomatöser, teils auch verruköser Tumor, der an der Oberfläche gelegentlich mit Krusten bedeckt sein kann infolge spontaner Fistulation oder Ulzeration bzw. mechanischer Irritation.

Histologisch sind für das SCAP die unter einer häufig akanthotischen und gelegentlich auch papillomatösen Epidermis im oberen und mittleren Korium gelegenen zysten- und gangartigen mit einem zweireihigen Epithel aus basal kuboiden, lumenwärts zylindrischen Zellen ausgekleideten Hohlräume

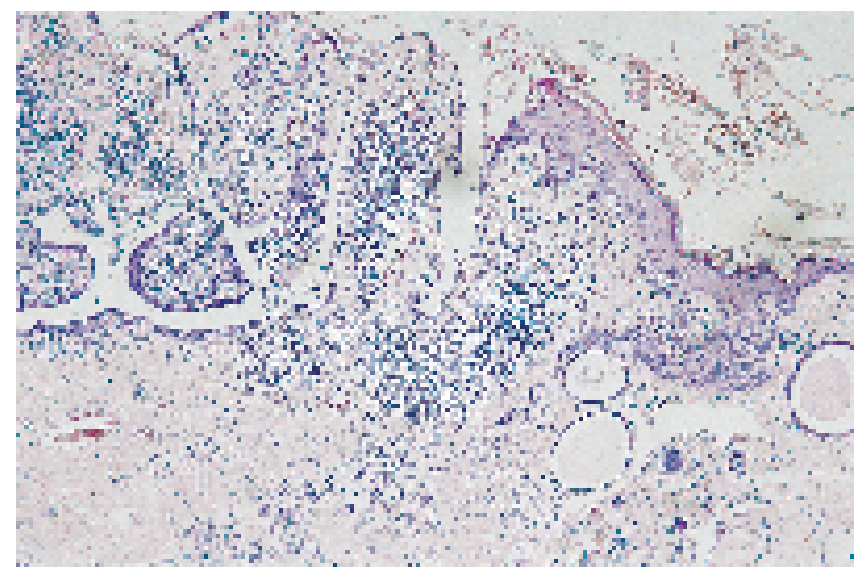

Abb. 2 SCAP und oberflächlich-solides Basalzellkarzinom, HE x 80 .

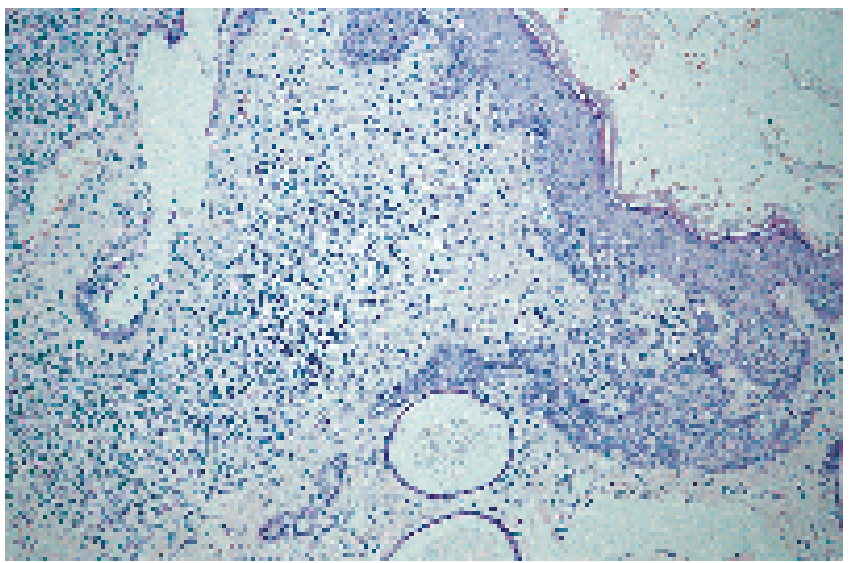

Abb.4 Oberflächlich-solides Basalzellkarzinom, HE x 125.

mit zottenförmigen Epithelproliferationen in diese hinein charakteristisch. Mündungen an der Oberfläche sowie Verbindungen der Zysten oder Gänge mit tief im Korium liegenden Schweißdrüsen sind ebenfalls typisch. Bei letzteren handelt es sich vorwiegend um apokrine Drüsen, und unter Berücksichtigung des entsprechenden Zystenepithels weisen diese Merkmale das SCAP als apokrinen Schweißdrüsen-Tumor aus $[11,14,19]$. Andererseits sind auch Differenzierungen in Richtung ekkriner Schweißdrüsen beschrieben worden [6, 19], so dass unter Umständen erst mittels immunhistochemischer Marker eine Zuordnung zum jeweiligen Sekretionstyp getroffen werden kann $[2,9,18]$. Das häufig nachgewiesene unterschiedlich stark ausgebildete plasmazelluläre Infiltrat des Tumorstromas wird auf sekundär chronisch entzündliche Veränderungen zurückgeführt $[6,11,15,19]$. In unserem Fall waren in einem vorwiegend lymphozytären Infiltrat nur einzelne Plasmazellen nachweisbar.

Vorrangig ist das SCAP auf dem Kapillitium sowie in der Stirnund Schläfenregion lokalisiert $[6,15,19]$. Weniger häufig wurde es in anderen Gesichtspartien [6,15,19], wie am Oberlid [1], an der Ober- [6] und Unterlippe [21] sowie im äußeren Gehörgang [16] beobachtet. Darüber hinaus ist sein Vorkommen an Hals, Brust, Rücken [6, 15, 19], in der Axilla [19], am Abdomen 
$[6,19]$, in der Leistenregion, am Gesäß, Perineum, Skrotum, Oberschenkel [6] und Unterschenkel $[15,17]$ beschrieben worden.

Ein bevorzugtes Auftreten des SCAP bei einem von beiden Geschlechtern läßt sich nicht eindeutig belegen $[15,19]$; ein Bericht über ein häufigeres Vorkommen bei Männern [6] ist am ehesten auf die untersuchte Patientenklientel (Militärangehörige) zurückzuführen.

Obwohl von etwa 50\% der Betroffenen ein Bestehen des Tumors seit der Geburt angegeben wird [6], liegt der Altersgipfel bei Diagnosestellung jenseits des 30. Lebensjahres [15, 19]. Diese Tatsache könnte zusammen mit klinisch-morphologischen und anamnestischen Faktoren auch der Grund dafür sein, dass das SCAP gelegentlich unter einer anderen Diagnose exzidiert wird $[1,21]$.

In $2-30 \%$ entwickelt sich das SCAP in einem Naevus sebaceus $[3,6,8,13-15]$ und kann unter diesen Bedingungen noch mit weiteren, vorwiegend benignen Tumoren $[3,15]$, aber auch mit einem Basalzellkarzinom vergesellschaftet sein $[6,8,12-15$, 22, 25, 26]. Dabei ist das Auftreten eines Basalzellkarzinoms in einer Kombination von SCAP und Naevus sebaceus mit 16 $26 \%$ sogar relativ häufig $[6,13,15]$. In der zahlenmäßig größten Untersuchungsserie zum SCAP mit insgesamt 100 Patienten von Helwig und Hackney [6] wiesen 34 gleichzeitig einen Naevus sebaceus auf, 9 davon (26\%) zusätzlich ein Basalzellkarzinom.

Im Gegensatz dazu war in unserem Fall das SCAP mit einem Basalzellkarzinom assoziiert, ohne gleichzeitig vorliegenden Naevus sebaceus. Eine solche Koinzidenz wird offenbar deutlich seltener angetroffen. Wir fanden diesbezüglich in der recherchierten Literatur nur drei einschlägige Berichte. So diagnostizierten Pinkus [19] unter 17, Mensing und Jänner [15] unter 11 Fällen jeweils ein SCAP, das mit einem Basalzellkarzinom vergesellschaftet war; Zugerman [28] beschreibt wie wir einen Einzelfall. Unter Berücksichtigung der verbleibenden 66 reinen SCAP-Fälle von Helwig und Hackney [6] lässt sich somit für die Kombination SCAP + Basalzellkarzinom eine Häufigkeit von $2 \%$ errechnen.

Bezüglich des histologischen Typs der mit einem SCAP kombinierten Basalzellkarzinome ist keine spezielle Dominanz zu erkennen. Es wurden oberflächliche und solide, teils pigmentierte Varianten beschrieben $[15,19,28]$. Jedoch repräsentieren nicht alle mit einem SCAP assoziierten basaloiden Strukturen tatsächlich ein Basalzellkarzinom. Es kann sich dabei auch um Trichoblastome handeln, die unter Umständen lichtmikroskopisch schwierig von Basalzellkarzinomen abzugrenzen sind $[10,23]$. Hier ist der immunhistochemische Nachweis von Merkel-Zellen hilfreich, die in Trichoblastomen stets vorkommen, in Basalzellkarzinomen bisher jedoch nicht gefunden wurden [4, 24]. Zur Markierung von Merkel-Zellen werden Antikörper gegen Zytokeratin-8, -18, -20, Chromogranin A und neuronspezifische Enolase mit Erfolg eingesetzt [4, 20, 24]. In unserem Fall waren in den basaloiden Tumorformationen keine NSE-positiven Zellen nachweisbar; der Tumor stellte somit ein Basalzellkarzinom dar.

Offensichtlich entwickeln sich das SCAP und das mit ihm vergesellschaftete Basalzellkarzinom unabhängig voneinander.
Eine maligne Entartung der präexistenten Adenomstrukturen wurde bisher nicht nachgewiesen [15, 19, 28], und auch bei unserer Patientin ergab sich dafür feingeweblich kein Anhalt. Wir fanden beide Tumoranteile eng beieinanderliegend vor. Nach diesem Befund dürfte es sich in unserem Fall am ehesten um einen Kollisionstumor handeln. Dabei haben möglicherweise die einzelnen Tumorkomponenten eine gemeinsame Histogenese. Basalzellen, Plattenepithel, Haare, Talg- und apokrine Schweißdrüsen entstammen dem primären Epithelkeim. Lever und Schaumburg-Lever [11] sehen das Basalzellkarzinom als Hamartom an, das sich ebenfalls aus Zellen des primären Epithelkeims herleitet. Demnach könnten das SCAP sowie ein mit ihm assoziiertes Basalzellkarzinom das Ergebnis von Proliferation und Entwicklung pluripotenter primärer Epithelkeimzellen in unterschiedliche Richtungen sein, ausgelöst durch noch unbekannte Stimuli $[11,25,28]$.

Wie bei unserer Patientin wurden eindeutige Kombinationstumoren aus SCAP + Basalzellkarzinom bisher ausschließlich im Kopfbereich nachgewiesen $[15,19,28]$. Das entspricht der Hauptlokalisation des SCAP.

In der Regel wird das mit einem SCAP kombinierte Basalzellkarzinom klinisch nicht diagnostiziert, sondern kommt erst bei der histologischen Untersuchung zur Darstellung. Auch wenn eine solche Koinzidenz offenbar selten vorliegt, sollte deshalb bei einem SCAP grundsätzlich aktiv therapeutisch vorgegangen werden. Die Therapie der Wahl ist eine frühzeitige Exzision mit entsprechendem dreidimensionalen Sicherheitsabstand.

\section{Literatur}

${ }^{1}$ Abanmi A, Joshi K, Atukorala D, Okla R. Syringocystadenoma papilliferum mimicking basal cell carcinoma. J Am Acad Dermatol 1994; 3: 127 - 28

${ }^{2}$ Ansai S, Koseki S, Hozumi Y, Kondo S. An immunohistochemical study of lysozyme, CD-15 (Leu M1), and gross cystic disease fluid protein-15 in various skin tumors: assessment of the specifity and sensitivity of markers of apocrine differentiation. Am J Dermatopathol 1995; 17: 249-255

${ }^{3}$ Chun K, Vazquez M, Sanchez JL. Nevus sebaceus: clinical outcome and considerations for prophylactic excision. Int J Dermatol 1995; 34: $538-541$

${ }^{4}$ Collina G, Eusebi V, Capella C, Rosai J. Merkel cells differentiation in trichoblastoma. Virchows Arch 1998; 433: 291 - 296

${ }^{5}$ Elliot GT. Adeno-cystoma intracanaliculare occuring in a naevus unius lateris. J Cut Genitourin Dis 1893; 11: 168 - 173

${ }^{6}$ Helwig EB, Hackney VC. Syringadenoma papilliferum. Arch Dermatol 1955; 71: $361-372$

${ }^{7}$ Jadassohn J. Bemerkungen zur Histologie der systematisierten Naevi und über „Talgdrüsennaevi”. Arch Dermatol Syph 1895; 33: $355-407$

${ }^{8}$ Jones EW, Heyl T. Naevus sebaceus: a report of 140 cases with special regard to the development of secondary malignant tumors. Br J Dermatol 1970; 82: 88 - 117

${ }^{9}$ Kanitakis J, Schmitt DL, Bernard A, Boumsell L, Thivolet J. Anti-D 47: a monoclonal antibody reacting with the secretory cells of human eccrine sweat glands. Br J Dermatol 1983; 109: 509 - 513

${ }^{10}$ Karnbach C, Neuber K, Diaz-Cascajo C, Steinkraus V. Syringocystadenoma papilliferum und Trichoblastom innerhalb eines Naevus sebaceus. Hautarzt 1998; 49: 654-656

${ }^{11}$ Lever WF, Schaumburg-Lever G. Histopathology of the skin. Ed 7. Philadelphia: Lippincott, 1990: 594-596 
${ }^{12}$ Lillis PJ, Ceilley RI. Multiple tumors arising in nevus sebaceus. Cutis 1979; 23: $310-314$

${ }^{13}$ Linse R, Hadlich J, Ehlers E, Schubert H. Sekundärproliferationen auf Naevus sebaceus. Dermatol Mschr 1987; 173: 153-157

${ }^{14}$ Mehregan AH, Pinkus H. Life history of organoid nevi. Arch Dermatol 1965; 91: $574-588$

${ }^{15}$ Mensing H, Jänner M. Naevus sebaceus Jadassohn und Syringocystadenoma papilliferum. Hautarzt 1981; 32: 130-135

${ }^{16}$ Müller R, Theissig F. Syringocystadenoma papilliferum des äußeren Gehörganges. Laryngo Rhino Otol 1995; 74: 43-45

${ }^{17}$ Ndiaye B, Kane A, Develoux M, Dieng MT, Saccharin C. Le syringocystadenome papillifere: un cas localise sur le genou. Ann Dermatol Venereol 1994; 121: 323 - 324

18 Paties C, Taccagni GL, Papotti M, Valente G, Zangrandi A, Aloi F. Apocrine carcinoma of the skin: a clinicopathologic, immunocytochemical, and ultrastructural study. Cancer 1993; 71: 375 - 381

19 Pinkus $\mathrm{H}$. Life history of naevus syringadenomatosus papilliferus. Arch Dermatol 1954; 69: 305 - 322

${ }^{20}$ Plettenberg A, Pammer J, Tschachler E. Merkel cells and Merkel cell carcinoma express the BCL- 2 protooncogene. Exp. Dermatol 1996; 5: $183-188$

21 Pullmann H, Pfeiff B. Syringocystadenoma papilliferum als Differentialdiagnose eines Unterlippencarcinoms. Z Hautkr 1984; 6: $348-355$

22 Rosenblum GA. Nevus sebaceus, syringocystadenoma papilliferum, and basal cell epithelioma. J Dermatol Surg Oncol 1985; 11: $1018-1020$

${ }^{23}$ Schirren CG, Rütten A, Sander C, McClain S, Diaz C, Kind P. Das Trichoblastom: Ein Tumor mit follikulärer Differenzierung. Hautarzt 1995; 46: $81-86$

${ }^{24}$ Schulz T, Hartschuh W. Merkel cells are absent in basal cell carcinomas but frequently found in trichoblastomas: an immunohistochemical study. J Cutan Pathol 1997; 24: 14-24

${ }^{25}$ Stavrianeas NG, Katoulis AC, Stratigeas NP, Karagianni IN, Paterou-Stavrianea M, Vareltzidis AG. Development of multiple tumors in a sebaceus nevus of Jadassohn. Dermatology 1997; 195: $155-158$

${ }^{26}$ Thiers H, Moulin G, Joseph JY, Rouhani A. Syringocystadenome papillifere et epithelioma basocellulaire sur naevus sebace de Jadassohn. Bull Soc Fr Dermatol 1969; 76: 369-371

${ }^{27}$ Werther L. Syringadenoma papilliferum (Naevus syringadenomatosus papilliferus). Arch Dermatol Syph 1913; 116: 865-870

28 Zugerman I. Basal cell epithelioma on nevus syringocystadenomatosus papilliferus. Arch Dermatol 1961; 84: 672 -675

\section{Dr. Michael Wiesner}

Klinik und Poliklinik für Hautkrankheiten der Ernst-Moritz-Arndt-Universität Greifswald

Fleischmannstraße 42 - 44

17487 Greifswald 